\title{
A strain space framework for numerical hyperplasticity
}

\author{
L.G. Margolin* \\ Los Alamos National Laboratory, Los Alamos, NM 87545, USA
}

\begin{abstract}
Numerical simulations of high strain rate plastic flow have historically been built in a hypoelastic framework and use radial return (Wilkins' method) as the solution algorithm. We show how each of these choices can lead to inaccurate and possibly nonconvergent results. We describe an alternative solution procedure based on a simple multiple time scale perturbation theory that is stable, accurate, computationally efficient and simple to implement. Further extension of these results then leads to a strain space formulation that has additional computational advantages. We illustrate our development with numerical experiments.
\end{abstract}

This paper is dedicated to my friend and colleague Christo Christov on the occasion of his 60th birthday, in recognition of his many important and creative contributions to the formulation of continuum mechanics.

Key words: Numerical plasticity, Hyperplasticity, Wilkins' method,

* Tel.: +1-505-665-1947; fax: +1-505-665-6722.

Email address: len@lanl.gov (L.G. Margolin).

Preprint submitted to Elsevier

12 April 2012

(C) 2012. This manuscript version is made available under the Elsevier user license http://www.elsevier.com/open-access/userlicense/1.0/ 


\section{Introduction}

In the early days of numerical simulation when computers were slow and memory limited, Mark Wilkins devised an accurate and efficient method for solving the equations of dynamic plasticity. First published in a Livermore technical report [22] in 1963 and the following year in volume 3 of Methods of Computational Physics [21], the method of radial return (often termed Wilkins' method) has been widely cited and is still in common use today. Wilkins' method uses a rate form of the equations in which incremental stress is calculated as a function of stress and rate of deformation; rate formulations in which stress is a history-dependent functional are termed hypoelastic $[20,3]$.

At roughly the same time, a theoretical framework for plasticity was being formulated $[23,15,17]$. Plastic flow is a dissipative process and irreversible thermodynamics is the proper context in which to describe it. The resulting theory, termed thermomechanics, is a hyperelastic theory, which derives from the assumption of a thermodynamic potential. In hyperelastic constitutive models, stress is a function of strain and internal variables which contain the history dependence.

An associated theoretical issue was raised by Naghdi and Trapp in 1972 [2,16], namely that plastic yield criteria needed to be formulated in strain space rather than in stress space. Among the issues they raise, at least one is of computational importance, that strain is not necessarily a unique function of stress.

Despite its long term success in the computational mechanics community, Wilkins' method has some shortcomings of both accuracy and stability, especially when applied to high strain rate phenomena. The accuracy issues arise from the explicit nature of Wilkins' method and can be readily mitigated by subcycling or implicit solution techniques - possibly at significant computational expense. The stability issues are more subtle, originating in the coupling of radial return with the momentum equation.

In previous work, Margolin and Flower [13] offered an alternative to Wilkins' method for high strain plasticity. The algorithm is based on multiple time scale perturbation theory and addresses issues of both accuracy and stability while preserving computational efficiency. This algorithm is still cast in the framework of hypoelasticity.

There are important advantages to the hyperelastic framework, both theoretical and computational. However, most of the development effort in the computational community has been devoted to improving the constitutive models and in particular to the formulation of yield surfaces in stress space. The implementation of a hyperelastic framework consistent with [16] would 
require that these models be converted from stress space to strain space and we speculate that this is one of reasons the computational community does not use hyperelasticity more widely.

The main purpose of this paper is to demonstrate how the perturbation approach of [13] can be extended to convert a stress-based hypoelastic model to a strain-based hyperelastic model for the simple case of a von Mises (constant) yield surface. In $\S 2$ we briefly review the equations of dynamic elasto-plasticity. In $\S 3$, we describe Wilkins' method and show that it is a first-order explicit approximation to the analytic equations. In $\S 4$ we offer numerical experiments to illustrate the potential inaccuracies and to identify the circumstances that are susceptible to error.

In $\S 5$ we describe a simple multiple time scale theory which leads to an analytic solution of the plasticity equations. The theory is based on two assumptions, namely that the strain rate can be considered approximately constant over a computational cycle, and that the total strain can be additively decomposed into an elastic component and a plastic component. These assumptions are generally valid and not restricted to the case of perfect plasticity.

In $\S 6$, we modify the multiple time scale theory to replace the yield condition in stress space with a constitutive relation for plastic strain. This represent a first step in creating a true hyperelastic framework that can consistently incorporate modern thermomechanical ideas. We conclude the paper with a discussion in $\S 7$ of the numerical advantages of our strain space formulation.

\section{Elastic-plastic equations}

Here, we briefly review the equations that describe time dependent elasticplastic flow. More detailed discussions and derivations can be found in many classic textbooks, e.g., in Hill [6], Khan \& Huang [8], Lubliner [9], etc. Also, many details of the numerical formulation and implementation of plasticity models can be found in Simo and Hughes [19].

Inelasticity in solids is often modeled computationally as plastic flow. In practical terms, finite material strength places limits on the size of the components of the deviatoric stress tensor $s_{i j}$ while dissipating kinetic energy. The total deviatoric strain, $\Delta e_{i j}=\dot{e}_{i j} \Delta t$ realized over some interval of time $\Delta t$, will be divided $^{1}$ between an elastic or reversible strain $\Delta e_{i j}^{e}$, and a plastic or irre-

1 Sometimes a multiplicative decomposition of the deformation gradient is assumed. However, as discussed in [1] and [4], the additive decomposition is to be preferred for finite strains whereas the two are equivalent for infinitesimal strains we assume. 
versible strain $\Delta e_{i j}^{p}$ :

$$
\Delta e_{i j}=\Delta e_{i j}^{e}+\Delta e_{i j}^{p}
$$

The plastic strain causes permanent changes in the reference (i.e., unloaded) state of the material. The associated plastic work contributes to the material temperature. However, some fraction of the plastic work is consumed in microstructural changes to the material rather than heat [18].

The onset of plastic flow is described by a yield condition that in numerical algorithms is usually formulated in stress space. Specifically, in rate independent (perfect) plasticity one specifies a function of the deviatoric stresses, usually the second invariant $J_{2}$, to be always less than or equal to the square of the yield strength $Y$

$$
J_{2} \equiv \frac{1}{2} s_{j k} s_{j k} \leq Y^{2} .
$$

The yield stress is a material property, and may also depend on the pressure, the temperature, the accumulated plastic strain or plastic work, and other internal variables. The yield surface is closed and convex. Conceptually, it divides the state space of stress deviators into three regions: inside the surface, the states are elastic, while on the surface the states are plastic. There are no physically attainable states outside the yield surface in an elastic perfectly plastic model. Then the mechanical behavior of an elastic-plastic material is described by three ingredients:

(1) the stress-strain relation in the elastic region,

(2) the yield condition that determines where plastic flow begins and ends,

(3) the stress-strain relation or flow law for the plastic region.

In the elastic region, the stress-strain relations for a linear, isotropic medium are

$$
\begin{aligned}
s_{j k} & =2 G e_{j k} \\
\sigma_{j j} & =3 K \epsilon_{j j} .
\end{aligned}
$$

In these equations, $G$ is the shear modulus and $K$ is the bulk modulus. Also, $\sigma_{j k}$ is the total stress tensor and $\epsilon_{j k}$ is the total strain tensor. All of the stress and strain tensors are symmetric in the indices $j$ and $k$. The shear modulus and the bulk modulus are related to Youngs' modulus E and Poisson's ratio $\nu$ :

$$
G=\frac{E}{2(1+\nu)} \quad ; \quad K=\frac{E}{3(1-2 \nu)} .
$$

The stress-strain relation for a state that begins on the yield surface depends on the loading. We define the work rate function $W$

$$
W \equiv s_{j k} \frac{d e_{j k}}{d t} .
$$


When $W \geq 0$, one is loading and so is remaining on the yield surface. In the simple case that the yield $Y$ is constant and the yield surface given by eq. (2), the stress rate equation is:

$$
\frac{d s_{j k}}{d t}=2 G\left(\frac{d e_{j k}}{d t}-\frac{W}{2 Y^{2}} s_{j k}\right) .
$$

On the other hand, when $W<0$ one unloads elastically:

$$
\frac{d s_{j k}}{d t}=2 G \frac{d e_{j k}}{d t}
$$

Note that eq. (6) ensures that the final state lies on the yield surface; i.e., contracting eq. (6) with $s_{j k}$ and using eqs. (2) and (5) leads to

$$
\frac{d}{d t}\left(s_{j k} s_{j k}\right)=0
$$

Furthermore, comparison with eq. (1) identifies the constitutive relation for plastic strain:

$$
\begin{array}{ll}
\frac{d e_{j k}^{p}}{d t}=\frac{W}{2 Y^{2}} s_{j k} & \text { if } W \geq 0 \\
\frac{d e_{j k}^{p}}{d t}=0 & \text { if } W<0 .
\end{array}
$$

This paper is mainly concerned with eq. (6) or equivalently eq. (8). In the next two sections, we will show how Wilkins' method approximates these equations and under what circumstances the approximation breaks down.

\section{Wilkins' method}

Perfect plasticity is an equilibrium constitutive model, i.e., the theory contains no physical time scales. However, a time scale does appear in numerical simulations, the time step of the calculation, $\Delta t$. Under some circumstances, particularly in high strain rate calculations, the numerical integration of the plastic stress-strain equations can be inaccurate and can lead to significant errors in the final stress state or in the apparent lack of convergence. The problem lies in the numerical approximation (i.e., Wilkins' method) that is used to integrate the plastic stress-strain relations. In the next few paragraphs, we review how the elastic-plastic equations are solved in many dynamic multidimensional stress wave codes that utilize an explicit time-marching procedure. Following common usage, we refer to the explicit solution of radial return as Wilkins' method. 
At the beginning of each computational cycle, the total strain rate tensor is constructed from the material velocities. This strain rate tensor and the stresses at the beginning of the cycle are then input to the constitutive model - in this case, the elastic-plastic model - and the updated stresses are calculated. The new stresses are then used in the momentum equation to update the material velocities, completing the cycle. That is, the constitutive model and the momentum equation are not iterated with each other to consistency. However, the computational time step $\Delta t$ is limited by a Courant-FriedrichsLevy (CFL) condition that ensures both the accuracy and stability of the momentum equation. We note that the CFL condition does not necessarily ensure the stability of the momentum equation coupled to the constitutive law; see [5].

The integration of the elastic-plastic equations within the constitutive model involves several steps. First, one assumes that all the strain is elastic and calculates a trial solution. The elastic trial solution for the deviators is given by

$$
s_{j k}^{*}=s_{j k}^{n}+2 G \dot{e}_{j k}^{n} \Delta t
$$

where $\dot{e}_{j k}^{n}$ is the deviatoric strain rate tensor. We use superscripts to indicate the discrete variables of a numerical simulation; e.g., superscript $n$ denotes the values at the beginning of the time level $n$ and the superscript $*$ indicates the elastic trial solution. Next, one compares the deviator stresses of the trial solution with the yield condition. If eq. (2) holds, then one accepts the trial solution and exits. Elsewise, one calculates one scalar factor to multiply each of the trial stress deviator components, which ensures that the final solution lies exactly on the yield surface.

$$
\begin{array}{rlrl}
s_{j k}^{n+1} & =s_{j k}^{*} & \text { if } & J_{2}^{*} \leq 2 Y^{2} \\
s_{j k}^{n+1}=s_{j k}^{*} \sqrt{\frac{2 Y^{2}}{J_{2}^{*}}} & \text { if } & J^{*}>2 Y^{2} .
\end{array}
$$

The explicit rescaling of the deviator stresses is called Wilkins' method [22] and has been in common use in solid dynamics codes like HEMP [21] and TENSOR [11] since the early 1960s.

It is not difficult to show that the method of radial return is an explicit, first-order approximation to the actual plastic stress-strain equations. Taylor expanding eq. (12) for 'small' $\Delta t$ shows that

$$
\frac{s_{j k}^{n+1}-s_{j k}^{n}}{\Delta t}=2 G\left(\dot{e}_{j k}^{n}-\frac{\left[s_{m n}^{n} \dot{e}_{m n}^{n}\right]}{2 Y^{2}} s_{j k}^{n}\right)+\mathcal{O}(\Delta t) .
$$

Here, 'small' means

$$
2 G\left\|\dot{e}_{j k}^{n}\right\| \Delta t<<\left\|s_{j k}^{n}\right\|
$$


that is, that the magnitude of the stress increment is much less than the magnitude of the stress itself. The problem is easily seen doing a scale analysis of eq. (14). Since $s_{j k}^{n}$ lies on the yield surface, it must have an order of magnitude less than or equal to $Y$. However, typical values for metals have a ratio,

$$
\frac{G}{Y} \sim 100
$$

So for Wilkins' method to give an accurate approximation, we must require

$$
\left\|\dot{e}_{j k}^{n}\right\| \Delta t<<.01
$$

On the other hand, stability of the momentum equation only requires a CFL condition of the form

$$
\frac{v \Delta t}{\Delta x}<1
$$

where $v=\sqrt{v_{j} v_{j}}$ is the material speed and $\Delta x$ is the size of a computational cell. Further,

$$
\left\|\dot{e}_{j k}^{n}\right\|=\frac{1}{2}\left\|\frac{\partial v_{j}}{\partial x_{k}}+\frac{\partial v_{k}}{\partial x_{j}}\right\| \leq \frac{v}{\Delta x} .
$$

In low strain rate processes, the inequality of eq. (15) above may satisfied. However, in high strain rate processes, and especially when shocks are present, steep gradients of velocity will be present and

$$
\frac{1}{2}\left\|\frac{\partial v_{j}}{\partial x_{k}}+\frac{\partial v_{k}}{\partial x_{j}}\right\| \sim \frac{v}{\Delta x} .
$$

In such a situation for accuracy reasons, one might need to reduce the computational timestep by order 100 or at least subcycle the Wilkins' method 100 times per computational cycle; in either case, substantially increasing the cost of the simulation. We will show that this is not necessary. However, before proceeding to the perturbation theory solutions, we will substantiate our scaling arguments in computational experiments.

\section{Computational experiments}

Here we investigate the accuracy of Wilkins' method. We will assume a starting state $s_{j k}^{n}$ that is on the yield surface. It is clear that Wilkins' method will be exact in the special case that the elastic test stress increment is in the same direction (in stress space) as the initial stress state, that is, if

$$
s_{j k}^{e}=2 G \dot{e}_{j k} \Delta t \sim s_{j k}^{n} .
$$

since in this case, the final state is the same as the initial state, $s_{j k}^{n+1}=s_{j k}^{n}$. Instead, we will assume that the elastic test stress increment is perpendicular. 


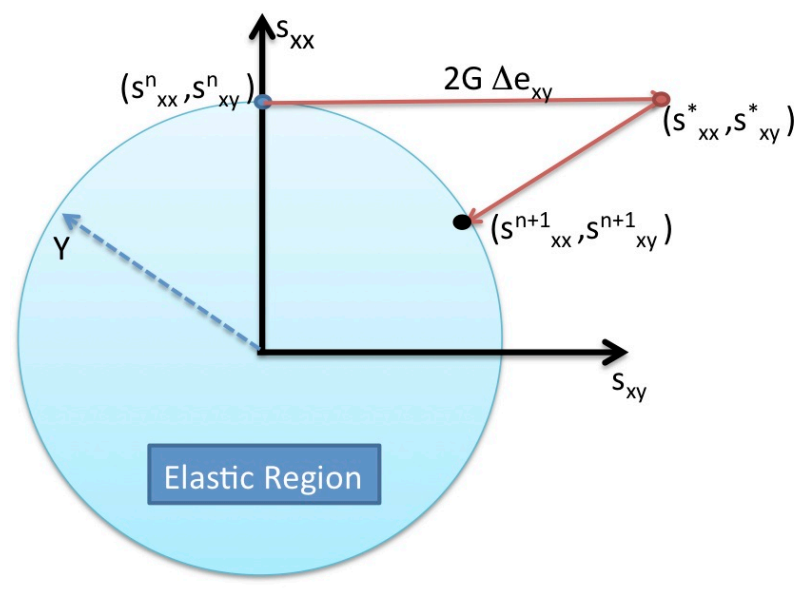

Fig. 1. Pictoral representation of Wilkins' method

We choose a typical ratio $G / Y \sim 100$ (the individual values do not matter), an initial state where $s_{x x}^{n}=\sqrt{2} Y$ and all other stress components are zero, and a strain rate where only $\dot{e}_{x y}$ is nonvanishing and constant. With these choices, only $s_{x x}^{n+1}$ and $s_{x y}^{n+1}$ will be nonzero. Since the final state must also lie on the yield surface, we can describe that state in terms of one variable, which we choose as

$$
\tan \psi=s_{x x}^{n+1} / s_{x y}^{n+1} .
$$

Figure 1 summarizes our experimental setups graphically in the $s_{x x^{-}} s_{x y}$ plane.

In these experiments, we will compare the solutions generated by radial return $(\tan \psi)$ with the exact solutions $\left(\tan \psi^{e}\right)$. The latter are found from the analytic solutions of $\S 5$. In the first experiment, we will vary the incremental strain $\dot{e}_{x y} \Delta t$ to identify where the error is greatest. In the second experiment, we will show how the error is reduced by subcycling - i.e., breaking the strain increment into smaller pieces.

We define the absolute error:

$$
\mathcal{E}_{a b s} \equiv\left|\tan \psi^{e}-\tan \psi\right|
$$

and the "elastic stress test increment"

$$
\Delta s_{x y}^{*}=2 G \dot{e}_{x y} \Delta t
$$

In Fig. 2 we plot the number of subcycles required to ensure $\mathcal{E}_{a b s} \leq .01$ This graph shows a maximum number of required subcycles (38) when the elastic stress test increment is of the same order as the yield stress.

$$
\left|\Delta s_{x y}^{*}\right| \sim Y
$$

This reflects the observation that as the magnitude of the elastic stress increment increases, the final state becomes less dependent on the initial state, 


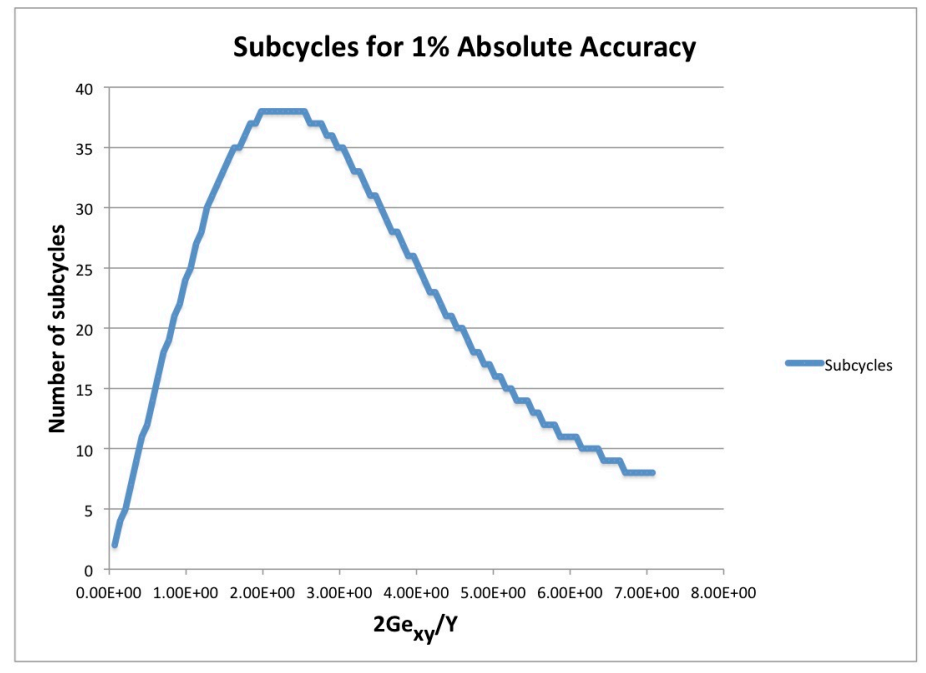

Fig. 2. Absoute error as a function of elastic stress increment

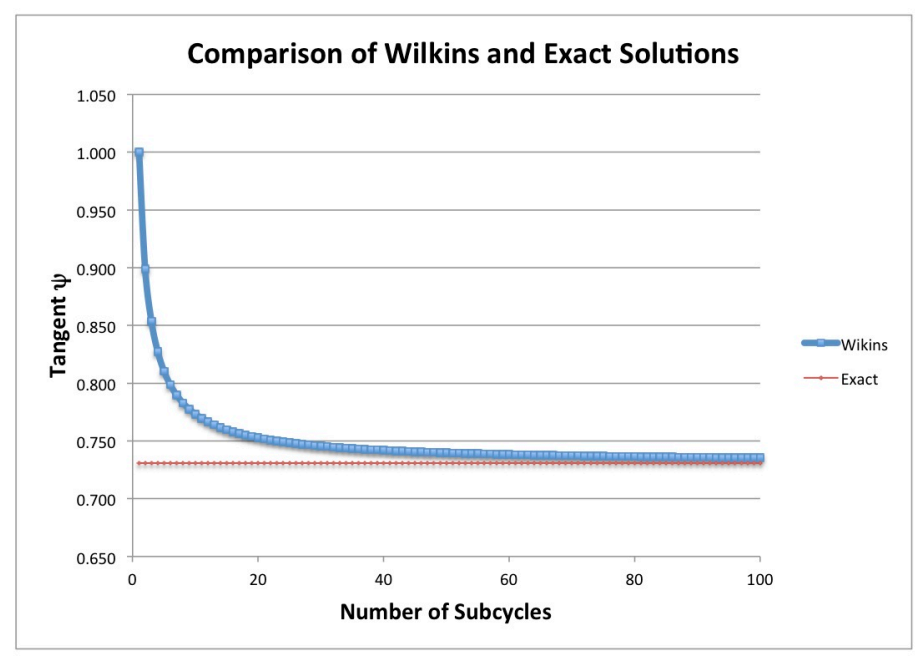

Fig. 3. Accuracy as a function of number of subcycles

while for small elastic stress increments Wilkins' method is an accurate approximation.

In Fig. 3, we specify $\Delta s_{x y}^{*}=Y$ and plot the Wilkins' method solution versus the exact solution as a function of the number of subcycles. Note that without subcycling, the error is almost 40\%. This figure illustrates another important point. The exact solution is found by subcycling eq. (21). Nevertheless, it is exactly constant - i.e., better than simply convergent. 


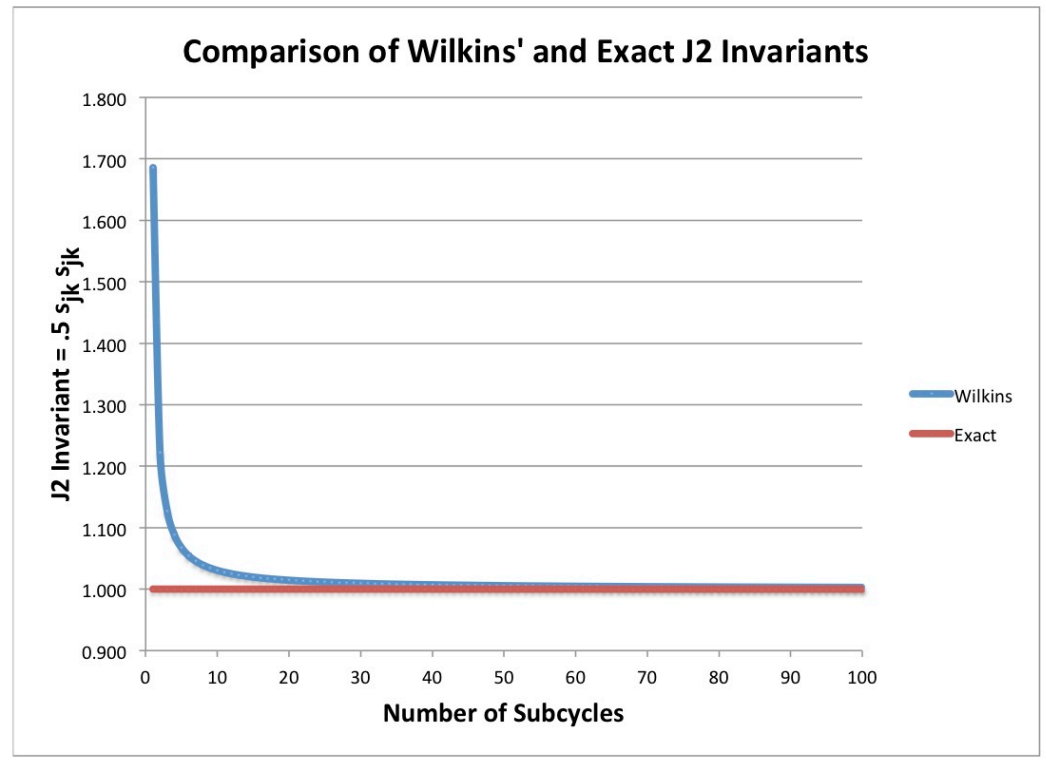

Fig. 4. Calculating the second invariant $J_{2}=.5 s_{j k} s_{j k}$ as a function of the number of subcycles. This is the same experiment as in Fig. 3, but using the strain space formulation with the integrals calculated from Wilkins' method.

\section{Stress increments for von Mises yield}

Equation (6) is a first-order differential equation for the stress deviators given the strain rate as a function of time and assuming a von Mises (i.e., a constant) yield surface. In an explicit dynamics computer program, the time step is controlled so that the strain rate may be considered constant over the cycle. This does not necessarily imply that the change in stress over the cycle is small, nor that the stress rate is approximately constant. For reasons discussed in the previous sections, an approximate solution of eq. (6) may not be satisfactory. The purpose of this section is to show that an exact solution is possible and is easily computed, assuming only that the strain rate is constant.

The first step is to contract eq. (6) with the strain rate tensor. Since each component is assumed constant in time, it may be brought inside the derivatives, yielding the following equation for the work rate $W$, defined in eq. (5)

$$
\frac{d W}{d t}=2 G\left(I^{2}-\frac{W^{2}}{2 Y^{2}}\right)
$$

Here,

$$
I^{2} \equiv \frac{d e_{j k}}{d t} \frac{d e_{j k}}{d t}
$$

is assumed to be a positive constant.

The solution to eq. (16) that satisfies the initial conditions $W(0)=W_{o}=$ 
$s_{j k}(0) \dot{e}_{j k}$ can be written

$$
W(t)=\frac{Y^{2}}{G} \frac{d}{d t}[\ln F]
$$

where

$$
F(t) \equiv A_{o} \exp (\alpha t)+\exp (-\alpha t)
$$

The constants in this expression are:

$$
\alpha \equiv \sqrt{2} I \frac{G}{Y} \quad ; \quad A_{o} \equiv \frac{\sqrt{2} I Y+W_{o}}{\sqrt{2} I Y-W_{o}}
$$

The second step is to substitute $W$ from eq. (17) into eq. (6). The equation for the deviator stresses now has the form

$$
\frac{d s_{j k}}{d t}=2 G \frac{d e_{j k}}{d t}-s_{j k} \frac{d}{d t}[\ln F]
$$

This is easily integrated with result

$$
s_{j k}(t)=s_{j k}(0) \frac{F(0)}{F(t)}+\frac{2 G}{F(t)} \frac{d e_{j k}}{d t} \int_{0}^{t} F\left(t^{\prime}\right) d t^{\prime} .
$$

Equation (20) is an exact solution for the deviator stress components as a function of time, assuming that the strain rate is constant.

For purposes of implementation, we think of this as an integral over a computational cycle, say from time $t^{n}$ to time $t^{n+1}$, then

$$
s_{j k}^{n+1}=s_{j k}^{n}\left[\frac{\chi_{n}\left(A_{n}+1\right)}{\left(A_{n} \chi_{n}^{2}+1\right)}\right]+\frac{\sqrt{2} Y}{I^{n}} \frac{d e_{j k}^{n}}{d t}\left[\frac{\left(A_{n} \chi_{n}^{2}-1\right)-\chi_{n}\left(A_{n}-1\right)}{\left(A_{n} \chi_{n}^{2}+1\right)}\right]
$$

where

$$
\chi_{n}=\exp \left(\alpha^{n} \Delta t\right) \quad ; \quad \alpha^{n} \equiv \sqrt{2} I^{n} \frac{G}{Y} \quad ; \quad A_{n} \equiv \frac{\sqrt{2} I^{n} Y+W^{n}}{\sqrt{2} I^{n} Y-W^{n}} .
$$

Also,

$$
I^{n} \equiv \sqrt{\frac{d e_{j k}^{n}}{d t} \frac{d e_{j k}^{n}}{d t}} \quad ; \quad W^{n}=s_{j k}^{n} \frac{d e_{j k}^{n}}{d t}
$$

It is simple to verify that the final state $s_{j k}^{n+1}$ lies exactly on the yield surface if the initial state does for any value of $\Delta t$. Squaring eq. (21) and assuming $s_{j k}^{n} s_{j k}^{n}=2 Y^{2}$ 


$$
\begin{aligned}
\left(A_{n} \chi_{n}^{2}+1\right)^{2} s_{j k}^{n+1} s_{j k}^{n+1} & =2 Y^{2} \chi_{n}^{2}\left(A_{n}+1\right)^{2} \\
& +2 \sqrt{2} \frac{W^{n} Y}{I^{n}} \chi_{n}\left(A_{n}+1\right)\left(A_{n} \chi_{n}^{2}-1\right) \\
& -2 \sqrt{2} \frac{W^{n} Y}{I^{n}} \chi_{n}^{2}\left(A_{n}^{2}-1\right) \\
& +2 Y^{2}\left[\left(A_{n} \chi_{n}^{2}-1\right)-\chi_{n}\left(A_{n}-1\right)\right]^{2} .
\end{aligned}
$$

Here, since $\chi_{n}=\exp \left(\alpha^{n} \Delta t\right)$ is an arbitrary number, the coefficients of $\chi^{m}$, $m=0 \ldots 4$ must each vanish separately.

$$
\begin{gathered}
\chi^{0} \cdot\left[s_{j k}^{n+1} s_{j k}^{n+1}-2 Y^{2}\right]=0 \\
\chi \cdot\left[-2 \sqrt{2} \frac{W^{n} Y}{I^{n}}\left(A_{n}+1\right)+4 Y^{2}\left(A_{n}-1\right)\right]=-0 \\
\chi^{2}\left[2 A_{n} s_{j k}^{n+1} s_{j k}^{n+1}-2 Y^{2}\left(A_{n}+1\right)^{2}+2 \sqrt{2} \frac{W^{n} Y}{I^{n}}\left(A_{n}^{2}-1\right)+2 Y^{2}\left(2 A_{n}-\left(A_{n}-1\right)^{2}\right]=0\right. \\
\chi^{3} \cdot\left[2 \sqrt{2} \frac{W^{n} Y}{I^{n}}\left(A_{n}\left(A_{n}+1\right)-4 Y^{2} A_{n}\left(A_{n}-1\right)\right]=0\right. \\
\chi^{4} \cdot\left[A_{n}^{2} s_{j k}^{n+1} s_{j k}^{n+1}-2 A_{n}^{2} Y^{2}\right]=0 .
\end{gathered}
$$

One can easily verify that all these equations will be individually satisfied if $s_{j k}^{n+1} s_{j k}^{n+1}=2 Y^{2}$. That is, the solution of eq. (21) lies exactly on the yield surface for any value of time increment.

Also for purposes of implementation, we note that eq. (21) represents integration along the yield surface; that is, we assume the initial point $s_{j k}^{n}$ lies on the yield surface. For the special case where the initial state lies inside the yield surface but the test solution $s_{j k}^{*}$ lies outside, one must break the time step in two separate time intervals. In the first interval, the stress trajectory is elastic until it first intersects the yield surface. In the second interval, the stress trajectory is constrained to move along the yield surface. If we denote the duration of the first interval by $f \Delta t$ where $f \in[0,1]$, then $f$ satisfies the quadratic equation

$$
(4 G I \Delta t)^{2} f^{2}+(4 G W \Delta t) f+\left(s_{j k}^{n} s_{j k}^{n}-2 Y^{2}\right)=0
$$

For the time interval $f \Delta t$, the elastic solution given in eqs. (10) and (11) is used, and subsequently for the time interval $(1-f) \Delta t$, the solution of eq. (21) is used. Although the case of loading from an elastic state to a plastic state requires this one-time special treatment, the case of unloading from a plastic state requires no special treatment; unloading is always elastic. 


\section{Strain space formulation}

Equation (21) represents considerable improvement over the standard Wilkins' method for the numerical integration of plastic flow for J2 flow theory; it is accurate, stable, and exactly convergent in the sense that is independent of subcycles. However, it is still in the form of a hypoelastic framework. Here, we reformulate our results from stress space to strain space, as a first step towards a true hyperelastic framework.

We formally integrate eq. (6) over the time interval $t \in[0, T]$, where $\mathrm{T}$ may be much larger than a computational time step:

$$
s_{j k}(t)=s_{j k}(0)+2 G\left\{\int_{0}^{T} \dot{e}_{j k} d t^{\prime}-\int_{0}^{T} \frac{\left(s_{m n} \dot{e}_{m n}\right)}{2 Y^{2}} s_{j k} d t^{\prime}\right\} .
$$

Now in the simulation, the integrals would be approximated as discrete sums over computational cycles $n \in[1, N]$. We write $\Delta t^{n}$ and $\dot{e}_{j k}^{n}$. to emphasize that we have made no assumption about the time step or the strain rate being constant from cycle to cycle,

$$
s_{j k}^{N}=s_{j k}^{1}+2 G\left\{\sum_{n=1}^{N} \dot{e}_{j k}^{n} \Delta t^{n}-\sum_{n=1}^{N} \mathcal{I}^{n}\right\} .
$$

Here

$$
\mathcal{I}^{n}=\frac{G}{Y^{2}} \int_{t^{n}}^{t^{n+1}}\left(s_{m n} e_{m n}\right) s_{j k} d t^{\prime}
$$

is an internal variable of the theory. That is, the first integrand can treated as a sum of constants whereas the second integrand must still be integrated over the individual computational cycles. These integrals are easily performed using eqs. (17) and (20) leading to

$$
\begin{aligned}
\mathcal{I}^{n} & =s_{j k}^{n}\left(1-\frac{\chi_{n}\left(A_{n}+1\right)}{A_{n} \chi_{n}^{2}+1}\right)+2 G \dot{e}^{n} \Delta t^{n} \\
& +\frac{2 G \dot{e}_{j k}^{n}}{\alpha^{n}} \frac{\chi_{n}\left(A_{n}-1\right)-\chi_{n}^{2} A_{n}+1}{A_{n} \chi_{n}^{2}+1} .
\end{aligned}
$$

Now we note that $s_{j k}^{1} / 2 G$ is the elastic strain of the initial state; likewise, $s_{j k}^{N} / 2 G$ is the elastic strain of state $t^{N}$. Then

$$
e_{j k}^{T} \equiv \frac{1}{2 G}\left(s_{j k}^{1}+2 G \sum_{n=1}^{N} \dot{e}_{j k}^{n} \Delta t^{n}\right)
$$


is the total strain of the cell at time $t^{N}$ and from eq. (1) we establish that

$$
e_{j k}^{P} \equiv \frac{1}{2 G} \sum_{n=1}^{N} \mathcal{I}^{n}
$$

is the total plastic strain. Thus eq. (25) can be written

$$
s_{j k}^{N}=2 G\left(e_{j k}^{T}-e_{j k}^{P}\right)
$$

which with the definitions of eqs. (27) and (28) constitute our strain space constitutive model.

Finally, we verify that the solution $s_{j k}^{N}$ lies exactly on the yield surface. In fact, it is simple to show that eqs. (25) and (26) are exactly equivalent to eq. (21) for one computational cycle. Then for each cycle,

$$
s_{j k}^{n} s_{j k}^{n}=2 Y^{2} \quad \Rightarrow \quad s_{j k}^{n+1} s_{j k}^{n+1}=2 Y^{2}
$$

(based on the proof at the end of $\S 5$ ), and so by induction, if $s_{j k}^{1}$ lies on the yield surface, then $s_{j k}^{N}$ must as well. The numerical experiment shown in Fig. 4 confirms this result.

\section{Discussion}

Equations (27), (28) and (29) are the main result of this paper. They represent an equation of state for stress as a function of total strain and plastic strain for the simple case of a von Mises yield condition. We recognize that a constant yield surface is too simple to be relevant for most applications. However, the ingredients in our formulation, namely the existence of multiple time scales and the additive decomposition of the total strain will apply to more general plasticity models.

Our formulation has many advantages over the commonly used solution technique (Wilkins' method) for numerical simulation. In $\S 3$, we identified situations where Wilkins' method could lead to large inaccuracies and in $\S 4$ we presented numerical experiments to quantify those inaccuracies. In particular, we showed that the largest errors may occur when the elastic stress test increment is of the same order as the yield stress - i.e., $\left|\Delta s_{x y}^{*}\right| \sim Y-$ a situation that is likely to occur in high strain rate deformations and when shocks are present. Our numerical experiments show that with sufficient subcycling, Wilkins' method asymptotically approaches the exact results with a consequent increase in computational effort and expense.

In $\S 3$, we also showed that Wilkins' method is an explicit approximation to the 
analytic model - i.e., to eq. (6) - which has the form of a Maxwell solid with variable relaxation time. Hicks [5] analyzes the numerical stability of explicit approximations to a Maxwell solid coupled with the momentum equation and shows that the coupled system will become linearly unstable when the effective relaxation time is less than the computational time step. The plasticity model will be nonlinearly stable by virtue of the yield condition which provides a quadratic upper bound on the size of the deviator stress components. However, the linear instability may be a source of noise in numerical simulations.

All of these issues can be avoided using the exact integrations of either $\S 5$ or $\S 6$ with essentially no additional computational effort or expense. However, the strain space formulation has an additional numerical advantage that we will describe briefly. High strain rate simulations are preferably made in Lagrangian codes where the mesh moves with the material so that one does not need to represent the advective terms. Since there is no conservation equation for stress in a hypoelastic framework, the treatment of the advective terms representing the flux of stress in any other reference frame is problematic. However, multidimensional Lagrangian codes are susceptible to mesh tangling, which reduces accuracy and often leads to premature termination of the simulation. One effective solution path is to employ an arbitrary LagrangianEulerian (ALE) code $[7,12]$ in which the mesh may be rezoned to preserve mesh quality and the solution remapped from the old mesh to the new mesh. One limiting case is to restore the mesh to its original configuration after every cycle; this technique of performing an Eulerian simulation is termed a continuous rezone.

Now the remapping stage is conceptually equivalent to advection where the mesh moves through the material rather than the material moving through the mesh [14]. Furthermore, the separation of a Lagangian stage where all of the dynamics is simulated and a remapping stage is an exact splitting of the equations. Thus, in the remapping stage, total strain and elastic strain are conserved, and so from eq. (1), plastic strain is conserved as well. Using our strain space formulation, one can readily remap total strain $e_{j k}^{T}$ defined in eq. (27) and total plastic strain $e_{j k}^{P}$ defined in eq. (28), and then reconstruct the stress field after remapping using eq. (29). This is entirely analogous to the remap process in fluids where energy and density are conserved quantities and can be remapped, and then pressure reconstructed from the equation of state.

We conclude this paper with these comments on rate independent (perfect) plasticity as a numerical model. Even though perfect plasticity has no physical time scales, our results show that it is a convergent model. Whereas Wilkins' method may require many subcycles or refinement of the computational time step for this convergence to become evident, our multiple time scale solutions show convergence for any time step properly limited by a CFL condition. On 
the other hand, the ever increasing size and speed of modern computers are now allowing one to resolve the physical time scales of rate dependent (viscoplastic) materials, so that perfect plasticity may not remain a viable physical model. Viscoplastic models are numerically stiff, so that explicit time integration is expensive and implicit time integration is typically used [10]. These are the same issues confronted in this paper for rate independent plasticity and so multiple time scale perturbation approach may provide a computationally efficient alternative for simulations of viscoplasticity as well.

\section{Acknowledgments}

The author gratefully acknowledges recent discussions with Randy Bos, Tom Dey and John Walter, and formative discussions in the past with Darryl Hicks and Paul Naghdi. The author also thanks two anonymous reviewers for their constructive suggestions. This work was performed under the auspices of the U.S. Department of Energy's NNSA by the Los Alamos National Laboratory operated by Los Alamos National Security, LLC under contract number DEAC52-06NA25396.

\section{References}

[1] J. Casey, P.M. Naghdi, A remark on the use of the decomposition $F=F_{e} F_{p}$ in plasticity, J. Appl. Mech. 47 (1980), 672-675.

[2] J. Casey, P.M. Naghdi, On the nonequivalence of the stress space and strain space formulations of plasticity theory, J. Appl. Mech. 50 (1983), 350-354.

[3] A.E. Green, Hypo-elasticity and plasticity, Proc. Royal Soc. London A234 (1956), 46-59.

[4] A.E. Green, P.M. Naghdi, Some remarks on elastic-plastic deformation at finite strain, Int. J. Engng. Sci. 9 (1971), 1219-1229.

[5] D.L. Hicks, Stability analysis of WONDY (a hydrocode based on the artificial viscosity method of von Neumann and Richtmyer) for a special case of Maxwell's law, Math. Comput. 32 (1971), 1123-1130.

[6] R. Hill, The Mathematical Theory of Plasticity, Oxford University Press (1998).

[7] C.W. Hirt, A.A. Amsden, J.L. Cook, An arbitrary Lagrangian-Eulerian computing method for all flow speeds, J. Comput. Phys. 14 (1974), 227-253.

[8] A.S. Khan,S. Huang, Continuum Theory of Plasticity, Wiley-Interscience (1995). 
[9] J. Lubliner, Plasticity Theory, Dover Publications (2008).

[10] A.M. Lush, G. Weber, L. Anand, An implicit time-integration procedure for a set of internal variable constitutive equations for isotropic elasto-viscoplasticity, Int. J. Plasticity 5 (1989), 521-549.

[11] B. Maenchen, S. Sack, The TENSOR code, in Methods in Computational Physics 3 (1964), B.A. Alder, S. Fernbach and M. Rotenberg, eds., Academic Press, NY, 181-210.

[12] L.G. Margolin, Introduction to "An arbitrary Lagrangian-Eulerian computing method for all flow speeds", J. Comput. Phys. 135 (1997), 198-202.

[13] L.G. Margolin, E.C. Flower, Numerical simulation of plasticity at high strain rate, ASME Materials D 26 (1991), 323-334.

[14] L.G. Margolin, M. Shashkov, Second-order sign-preserving conservative interpolation (remapping) on general grids, J. Comput. Phys. 184 (2003), 266298.

[15] P.M. Naghdi, A critical review of the state of finite plasticity, J. Appl. Math. Phys. (ZAMP) 41 (1990), 316-394.

[16] P.M. Naghdi, J.A. Trapp, The significance of formulating plasticity theory with reference to loading surfaces in strain space, Int. J. Engng. Sci. 13 (1975), 785797.

[17] P. Perzyna, The constitutive equations for rate sensitive plastic materials, Quart. Appl. Math. 20 (1963), 321-332.

[18] H.L. Schreyer, P.J. Maudlin, Thermodynamically consistent relations involving plasticity, internal energy and thermal effects, Phil. Trans. R. Soc. A363 (2005), $2517-2541$.

[19] J.C. Simo, T.J.R. Hughes, Computational Inelasticity, Springer (1998).

[20] C. Truesdell, Hypo-elasticity, J. Rat. Mech. Anal. 4, (1955), 83-133.

[21] M.L. Wilkins, Calculation of elastic-plastic flow, in Methods in Computational Physics 3 (1964), B.A. Alder, S. Fernbach and M. Rotenberg, eds., Academic Press, NY, 211-262.

[22] M.L. Wilkins, Calculation of elastic-plastic flow," Lawrence Livermore National Laboratory report UCRL-7322 (1963).

[23] H. Ziegler, C. Wehrli, The derivation of constitutive relations from the free energy and the dissipation function, Adv. Appl. Mech. 25 (1987), 183-238. 